(c) (i) (O)

\title{
Propuesta de un modelo de gestión para la docencia: experiencia de una universidad estatal chilena
}

\author{
FRANCISCO VILLEGAS VILLEGAS* \\ CLAUDIA VALDERRAMA HIDALGO**
}

Artículo de investigación sobre un modelo de gestión para la docencia universitaria.

Recibido: 8 de mayo de 2020 - Evaluado: 31 de julio de 2020 - Aceptado: 16 de septiembre de 2020

Citar como: Villegas, F. y Valderrama, C. (2021). Propuesta de un modelo de gestión para la docencia: experiencia de una universidad estatal chilena. Hallazgos, 18(35), 149-179. https://doi. org/10.15332/2422409X.5848

* Doctor en Didáctica por la Universidad de Antofagasta, Chile Correo electrónico: francisco.villegas@uantof.cl ORCID: https://orcid.org/0000-0001-8059-4299

** Máster en Educación por la Universidad de Antofagasta, Chile. Correo electrónico: claudia.valderrama@uantof.cl ORCID: https://orcid.org/0000-0003-1291-1816 


\section{Resumen}

Este artículo problematiza un tema relevante de la educación superior que entiende la gestión de la docencia como la capacidad de abordar los requerimientos actuales de la actividad docente universitaria en un contexto profesional. Para ello, y tomando como referencia las directrices de un proyecto ministerial, se expone la propuesta realizada en una universidad estatal chilena. De acuerdo con la información disponible, los docentes inician un tránsito hacia las funciones académicas, teniendo en cuenta las orientaciones de los tres componentes de calidad: gestión curricular y desarrollo docente, trayectoria del estudiante y gestión e innovación docente. En el enfoque de referencia se describen las relaciones entre docencia universitaria, enfoque curricular y gestión de la docencia. En el abordaje metodológico se presenta una idea de integración para la actividad formativa a través del problema que se va a resolver y el procedimiento utilizado, los logros obtenidos y las enseñanzas aprendidas para la gestión de la docencia. La experiencia demuestra los resultados a través de una descripción de la propuesta basada en los dos ejemplos de casos. Con base en estos se argumenta la definición de la gestión en innovación y el aseguramiento de la calidad curricular.

Palabras clave: Educación superior; Docencia efectiva; Gestión de la docencia; Política institucional. 


\title{
Proposal of a management model for teaching: experience of a Chilean state university
}

\begin{abstract}
This article problematizes a relevant issue in higher education that understands teaching management as the ability to address the current requirements of university teaching activity in a professional context. To this end, and taking as reference the guidelines of a ministerial project, the proposal made in a Chilean state university is presented. According to the available information, teachers start a transition towards academic functions, taking into account the orientations of the three quality components: curricular management and teacher development, student's trajectory and teaching management and innovation. In the reference approach, the relationships between university teaching, curricular approach and teaching management are described. The methodological approach presents an idea of integration for the training activity through the problem to be solved and the procedure used, the achievements and lessons learned for teaching management. The experience demonstrates the results through a description of the proposal based on the two case examples. Based on these, the definition of innovation management and curricular quality assurance is discussed.
\end{abstract}

Keywords: Higher education; Effective teaching; Teaching management; Institutional policy.

\section{Proposta de um modelo de gestão para a docência: experiência de uma universidade estadual chilena}

\section{Resumo}

Este artigo problematiza um tema relevante do ensino superior que entende a gestão da docência como a capacidade de abordar os requerimentos atuais da atividade docente universitária num contexto profissional. Para isso, e tendo como referência as diretrizes de um projeto ministerial, é apresentada a proposta realizada em uma universidade estadual chilena. De acordo com a informação disponível, os docentes iniciam uma transição às funções acadêmicas considerando as orientações dos três componentes de qualidade: gestão curricular e desenvolvimento docente, trajetória do estudante e gestão e inovação docente. $\mathrm{Na}$ abordagem de referência, são descritas as relações entre docência universitária, abordagem curricular e gestão da docência. Na metodologia, é apresentada uma ideia de integração para a atividade de formação por meio do problema a ser resolvido e o procedimento utilizado, as conquistas obtidas e as aprendizagens para a gestão da docência. A experiência demonstra os resultados por meio de uma descrição da proposta baseada nos dois exemplos de caso. Com base neles, a definição da gestão em inovação e a garantia da qualidade curricular são argumentadas.

Palavras-chave: Ensino superior; Docência efetiva; Gestão da docência; Política institucional. 


\section{Introducción}

En el ámbito actual del quehacer de las universidades estatales, tanto a escala internacional como en Chile, una de las acciones estratégicas más relevantes ha sido transitar hacia entornos cada vez más funcionales y dinámicos en el contexto de la gestión de la docencia universitaria, y cada vez más crecientes y profesionales, que permitan asumir una línea heterogénea, diversa y en plena construcción. Es lo que se ha llamado en la literatura especializada asumir el cambio profundo en múltiples dimensiones o mirada polifactorial, según lo indica Cole (2009). El presente artículo, por tanto, determina una propuesta que está orientada para una universidad estatal que pretenda definir estrategias y emprender acciones que permitan la gestión del currículo en sus dos aspectos esenciales: el desarrollo curricular y la acción pedagógica de la docencia, teniendo en cuenta que la educación puede desempeñar, a modo de lo que menciona Apple (2018), un rol sustantivo que desafíe situaciones en el contexto de la educación superior.

Con el propósito de ahondar en esta descripción, y en razón de los lineamientos institucionales de una universidad estatal que comienza en el modelo educativo, se profundiza en el tema de la promoción de las condiciones para el desarrollo docente universitario, con el fin de propiciar espacios que se ocupen de la innovación docente y la optimización de una serie de procesos que se encargan del estudiantado en su itinerario formativo. De este modo, se hace efectiva la triada de gestión docente, calidad y equidad e inclusión. En el esquema actual, y considerando lo relevante que es un impulso estratégico en una universidad para asegurar los éxitos de los estudiantes y, por otro lado, la conducción institucional de una organización de educación superior, el debate se caracteriza por las repercusiones en la principal función de las instituciones de educación superior en Chile: la docencia (Bernasconi, 2015). El ejercicio que están asumiendo dichas instituciones, en general, constituye un tema central al vincular y desarrollar óptimas propuestas ${ }^{1}$ de gestión docente de calidad ${ }^{2}$

1 Para el caso de este trabajo, la denominación de propuesta se asume como aquella consideración escrita que proporciona y determina para el usuario una información vinculada a todo lo que considere importante o relevante, con el propósito de tomar una decisión de la forma más eficiente y en grado de acción oportuna.

2 De acuerdo con Lemaitre et ál. (2012), "la calidad se puede entender como la coherencia entre fines institucionales, objetivos estratégicos y actividades que se realizan para cumplir con esos objetivos, considerando además los medios que se emplean para ello" (p. 27). 
en virtud de los desafíos permanentes que asume todo componente educativo universitario en el ámbito de los temas esenciales que hacen comprender la lógica de una universidad de avanzada, o bien en línea hacia una universidad compleja.

En ese sentido, los elementos de naturaleza docente, la descripción de las funciones de las unidades para dicho quehacer, las coberturas de los distintos agentes educativos, el desarrollo del propio docente como un comunicador que crea distintas relaciones entre él y los aprendices (Hernández y Guárate, 2017) y la gestión de la innovación e investigación de la práctica docente, entre otros aspectos, crean la necesidad de experimentar nuevos desarrollos que definan las transformaciones y exigencias de la educación superior. Las opiniones que se mencionan en este tema forman parte del reconocimiento de la legítima contribución de una entidad estatal como identificación de ese fundamento, en el sentido de asumir la visión representativa de los actores de la educación terciaria, que sirven al propósito de transformar los núcleos de prácticas docentes y de focalizar esfuerzos en las capacidades e innovaciones sostenibles en la universidad.

Para establecer el marco general de esta propuesta, se han determinado tres líneas de acción que cumplimentan dimensiones de transversalidad, las cuales pueden vincularse con la tributación de las suficiencias académicas y de gestión hacia la docencia universitaria, entendida esta como una responsabilidad institucional, según Paricio (2019), por el desarrollo de las capacidades de sus integrantes en el ámbito del proceso docente y formativo:

1. Articular una línea positiva de capacidades para facilitar una responsabilidad en la calidad del itinerario formativo del estudiantado, teniendo en cuenta el nuevo escenario por la pandemia a escala mundial.

2. Robustecer la gestión de la docencia, a través de una propuesta, asumiendo como foco de interés los procesos de aseguramiento interno de la calidad del proceso formativo.

3. Fortalecer en el profesorado los procesos de apoyo en la docencia que contribuyan a resultados de mediano y largo plazo, consistentes con los propósitos de la institución universitaria en su mirada de enfoque estratégico. 


\section{Marco de referencia y contexto de la propuesta}

\section{La docencia universitaria y un enfoque curricular ampliado}

La evolución de las organizaciones y el cambio permanente de las condiciones externas ejercen cada vez más presión sobre las organizaciones, lo cual las obliga a adecuarse constantemente a las nuevas condiciones. Esta sociedad se caracteriza por la transformación del aprendizaje, pues ya no solo basta saber cosas o cómo acceder a la información, sino que lo que se busca identificar es cómo abordar las situaciones complejas que se presentan. Debido a este carácter, los cambios para un desarrollo docente se configuran adecuadamente con miras a la orientación, la implementación de acciones y el seguimiento y apoyo en la práctica de aula del docente. En ese sentido, lo que nos recuerda Pimienta (2012) es dable de ser asumido por el profesorado en razón del enfoque de formación curricular centrado en el estudiante, del cambio de paradigma en la práctica educativa en el contexto de educación superior y el desarrollo de competencias ${ }^{3}$ para implementar situaciones de aprendizaje.

Ese contexto de cambio permanente, y altamente dominado por la incertidumbre, representa un proceso de trasformación para el sistema educativo en general, así como para las instituciones universitarias. En estas relaciones, tal como señala Bambrick-Santoyo (2018), para que la educación sea efectiva debe darse en el contexto de una gran enseñanza, es decir, mediante la presencia de una enseñanza de alta calidad, lo que recae en responsabilidades de gestión desde las propias organizaciones educativas.

En términos generales, en este escenario la propuesta parte del lugar que ocupa el conocimiento en la vida de los estudiantes y de cómo aquello es observado por la institución universitaria y por los docentes, según lo que se ha operacionalizado como un aprendizaje que merece la atención para el estudiantado desde un juicio cualitativo amplio (Perkins, 2016). Producto de lo anterior, se agrega un elemento extensivo en el campo de la gestión docente de progresiva evolución: amplificar el circuito curricular con nuevos desafíos y procesos, como en el caso de la inclusión de los nuevos contextos que ha obligado a los cierres temporales de las universidades a

3 Competencia, según Pimienta (2012), es un término polisémico, en que por consenso se entiende como el desempeño o la actuación integral de una persona, lo que implica conocimientos declarativos, habilidades, destrezas, actitudes y valores dentro de un contexto ético. 
raíz de la pandemia de la covid-19, ya que cerca de 25 millones de personas, entre estudiantes y profesores en América Latina y el Caribe, han dejado de asistir de manera presencial a los campus universitarios (Unesco/Iesalc, 2020).

En este sentido, cobra especial atención lo que indican Hax y Ugarte (2014) cuando expresan que es posible aspirar a una gran universidad, en el contexto de la docencia, que pretenda identificar dificultades y emprenda estrategias para el cambio. De este modo, si se aumentan las capacidades de consolidar y activar conocimientos, Chile tendrá un plus fundamental: asumir heterogeneidad en la manera de ver el futuro, y adaptarse a las variables que determina el sistema de educación superior de alta categoría, que incluye el desafío de introducir una perspectiva realista, académica y sistémica.

A lo anterior hay que agregar el tema particular del actual contexto de la pandemia por la covid-19, que para las organizaciones de educación superior, según el reporte de Reimers y Schleicher $(2020)^{4}$, implica asumir una oportunidad para que los líderes de dichas unidades educativas terciarias, teniendo en cuenta las propias instituciones, configuren una estrategia innovadora e intencionada para la prestación de los servicios educativos. En este marco referencial queda la sensación de que las necesidades de innovación en la gestión de la docencia y en el conocimiento curricular también son una aspiración conducente a recalcar y complejizar el quehacer, porque las universidades tienen que ampliar sus miradas de desafíos, utilizando variados enfoques estratégicos y mecanismos articuladores en la configuración de nuevas decisiones para la cultura docente universitaria. El contexto nacional y particular de la institución indica que son influjos para las decisiones del tipo de enseñanza que se imparte, la cual busca responder de manera pragmática a los diversos programas y planes de formación.

En este contexto, se entiende que las universidades que forman profesionales de diversas áreas del conocimiento dependen también del trabajo crucial de su profesorado. Por eso, consecuentemente, el abordaje consiste en dedicar más relaciones, nexos, trabajos conjuntos y cuidado en la propia formación docente, según lo indican Véliz y Bernasconi (2019). Esa variable comparte un enfoque de lo que significa asumir una tendencia global en el marco de un desarrollo de la gestión del profesorado

4 El informe, basado en una evaluación de las necesidades educativas y las respuestas emergentes en 98 países, identifica las prioridades más importantes que deben abordar en razón de la docencia y de la prestación de servicios educativos, así como del abordaje oportuno para enfrentar desafíos en la implementación de la educación en modalidad on line. 
basado en el perfeccionamiento activo y en la calidad entre la práctica docente, la investigación y la gestión de la docencia.

De esta manera, y de acuerdo con estudios como el de Santelices, Catalán y Horn (2018), las instituciones universitarias se proponen transitar hacia enfoques docentes y curriculares más integrales que indaguen y construyan una serie de factores. Entre estos se pueden mencionar los atributos de la misma institución de educación superior, las adaptaciones de las clases planificadas - incluyendo las herramientas virtuales o modalidades a distancia-, los recursos disponibles y la información sobre múltiples variables para el proceso de una toma de decisiones que identifique las prioridades de ese conocimiento por la docencia de calidad.

Con una evidente relación a lo mencionado por Véliz y Bernasconi (2019), estas consideraciones desarrollan una identificación todavía más reflexiva y crítica respecto de la importancia sobre temas específicos de la docencia universitaria, en tanto respuesta a la cuestión de qué significa una buena docencia en la educación superior, que incluye, entre otros aspectos, énfasis en el aprendizaje, el profesorado como tutor y facilitador, el fomento de las competencias y el docente como partícipe activo en programas de formación y actualización.

\section{La calidad hacia un nuevo modelo de gestión de la docencia}

Un asunto relevante que se identifica en los estudios de gestión de la docencia son las problemáticas que indican el aseguramiento de la calidad como un bien público y también en virtud de las relaciones entre la universidad con la sociedad. Por ejemplo, el trabajo de Morales y Rueda (2019) aporta evidencias acerca de que la calidad es la preocupación dominante en el actual debate de educación superior, así como en el futuro, puesto que hay factores que influyen en la calidad desde una línea sistémica y que correlacionan con las actividades de gestión en las universidades. Esto se puede traducir como tareas inconmensurables por la garantía pública con que desarrollan sus distintas funciones. Por esa razón, es ineludible evaluar, de manera permanente pero no de forma endogámica, la calidad de los procesos de formación en las diversas carreras y programas que la universidad ofrece, atendidas tanto por las definiciones de política estatal en relación con el cumplimiento de criterios de pertinencia, relevancia, eficiencia y eficacia que se exigen al sistema, como de compromisos institucionales que aspiran a la calidad total de los procesos que conducen a la formación profesional y de grado, en particular de los estudiantes de la propia institución.

La calidad debe ser definida y medida si se quiere mejorar. Es lo que indica, de manera crítica, Yao (2016) cuando analiza que la calidad, en el marco de la docencia, 
surge como un proceso de reflexión y de análisis. Sin embargo, el desarrollo de la calidad en el contexto de la organización universitaria permanece en tensión, debido a las intensificaciones del quehacer de gestión, ya que el proceso gradual de implementación guarda relación con una abundante burocracia y normativa que, en múltiples ocasiones, entran en directa colisión con los servicios que se entregan desde las unidades académicas.

Por otra parte, a escala global, cuando en la universidad se apunta, desde una perspectiva general, a la evaluación de la calidad de los procesos de formación, se está haciendo hincapié en la progresión y los resultados de lo que logran los estudiantes. En una clara relación con esto, las instituciones reconocen dos formas de evaluación de dicha calidad en el contexto del actual sistema nacional de aseguramiento de la calidad de la educación superior en Chile. En primer lugar se encuentra la evaluación externa, que implica la evaluación de la calidad de los procesos de formación, que está asociada a la acreditación de carreras y programas a cargo de una institución de carácter nacional: la Comisión Nacional de Acreditación (CNA) ${ }^{5}$, y está definida por procesos, criterios y estándares comunes para todas las instituciones (Ley 21.091 de 2018). Este quehacer se relaciona con varios procesos como la autoevaluación institucional, la visita de pares evaluadores y la emisión de juicio público — con determinación de años de acreditación—, que son parte de un sistema general nacional de gestión de la calidad de los procesos de formación profesional y de grado en las universidades chilenas.

En segundo lugar, en un nivel interno o propiamente institucional, es la universidad la que debe gestionar la calidad de los procesos asociados a la docencia de pre-y posgrado. Un insumo importante para incentivar cambios en este sentido es el proceso de autoevaluación que antecede a la acreditación, en el marco del juicio crítico que la propia institución debe levantar sobre las brechas entre lo que es y lo que quiere llegar a ser, para luego, tras el proceso de acreditación, abocarse a los procesos de monitoreo de las tareas de remediación sugeridas por los evaluadores externos, lo que implica asumir la práctica activa de los planes de mejora con sus referencias comparadas, observaciones generales y tendencias en los resultados e indicadores institucionales.

5 El quehacer de esta comisión está refrendado por lo que indica la Ley 20.129 de 2006, que establece un Sistema Nacional de Aseguramiento de la Calidad de la Educación Superior. 
De otro lado, y de forma particular, el estudio de Huerta y Gaete (2018) menciona que las políticas de calidad se encuentran en un lugar central en las universidades, las cuales tienen una dependencia organizacional de diversas unidades a cargo del proceso de aseguramiento de la calidad y de los procesos de acreditación. En este sentido, queda la sensación de que la acreditación y la autoevaluación representan así momentos o ciclos siempre tensionantes y críticos para la gestión, que buscan promover en las instituciones una cultura de mejoramiento continuo, afincada en la reflexión de aprendizaje y cambio orientado en la innovación y valoración cuidadosa de la planificación estratégica; aunque es necesario indicar las diferencias que se provocan en una institución por la complejidad y la diversidad de factores: la cultura interna, el contexto territorial, las creencias del profesorado, las necesidades del profesorado, las necesidades generales y globales de diversos actores, entre otros.

El Sistema Interno de Aseguramiento de la Calidad del Proceso Formativo, que tiene su correlato con el Sistema Nacional de Aseguramiento de la Calidad de la Educación Superior (Sinaces), establecido a través de una normativa de cobertura nacional (Consejo Nacional de Educación [CNED], 2017), descansa en tres componentes fundamentales: los estudiantes, los docentes y los sistemas de apoyo a los procesos formativos. Cada uno de estos componentes remite, a su vez, a un conjunto de procesos que deben ser considerados de manera integrada y compleja. En lo correspondiente a los estudiantes, que son el foco de un modelo educativo institucional centrado en una formación que despliega las competencias requeridas para el siglo XXI, el aseguramiento de la calidad del proceso formativo debe considerar el cómo la institución se hace cargo de los compromisos contraídos con ellos por carreras y programas. Estos incluyen la formación de un conjunto de competencias y capacidades durante un proceso de formación que debe ser de excelencia. Sin embargo, si se reflexiona en torno al sistema de aseguramiento de la calidad, se requieren variados elementos de supervisión interna y externa, con claro hincapié en la lectura de sus criterios y normas, así como de la identificación de los objetivos que la institución determine, acompañado de la idea de que la academia puede y debe ser mejor.

En relación con los docentes, que conforme a los requerimientos de los nuevos paradigmas deben crear las condiciones para la implementación efectiva en aula de los modelos educativos institucionales, el aseguramiento de la calidad del proceso formativo debe considerar el cómo la institución se hace cargo de generar las condiciones para lograr la creciente "profesionalización” de los docentes. De esta manera se asegura que el trabajo de aula permita mejorar los aprendizajes de los estudiantes y producir innovación tanto como investigación en docencia terciaria. 


\section{Experiencias de integración y de apoyo académico}

En lo concerniente a las experiencias de integración social y académica en la institución, la evidencia indica que se encuentran asociadas a programas de acercamiento con los establecimientos de enseñanza media, para apoyar e incluir estratégicamente un número de estudiantes a través de una gestión por la equidad en la educación superior. En este sentido, se evidencian programas institucionales que permiten atraer talentos a la universidad, como la asunción del sistema propedéutico, el programa de acompañamiento y acceso efectivo a la educación superior (PACE) y la nivelación para el ingreso a la vida académica. Estos esfuerzos aparecen como desafiantes, y contribuyen a la constitución de una experiencia de integración. Desde esta perspectiva, el diseño y la cobertura de acceso a la universidad han tenido niveles de importancia e influencia para la contribución a mejorar la calidad de vida de los estudiantes.

Hay avances significativos en estos procesos, aunque, como se desprende de lo que indican Santelices, Catalán y Horn (2018), estos programas en la institución estatal en comento aún trabajan con un número limitado de estudiantes, y su efectivo impacto debe ser estudiado en la composición total estudiantil. Por este motivo es algo pendiente en función de sus conclusiones.

Si bien el intento por generar un apoyo para estos espacios está encaminado, un elemento importante tiene que ver con los sistemas de apoyo al proceso formativo que se relacione con el desarrollo intelectual, el acompañamiento de los programas y los niveles de retribución para la calidad de los aprendizajes. En sintonía con lo anterior, esos apoyos académicos deben tener mayores niveles de eficiencia y eficacia en el quehacer institucional, y conformar un patrón de diseño y apoyo financiero en el contexto de la docencia, en virtud de programas, ayudantías, tutorías, entre otros, como experiencias de integración formativa. A continuación, la figura 1 presenta los elementos concernientes para el aseguramiento de la calidad en función del proceso de formación, con la intención de cumplir los objetivos institucionales de una universidad estatal. 


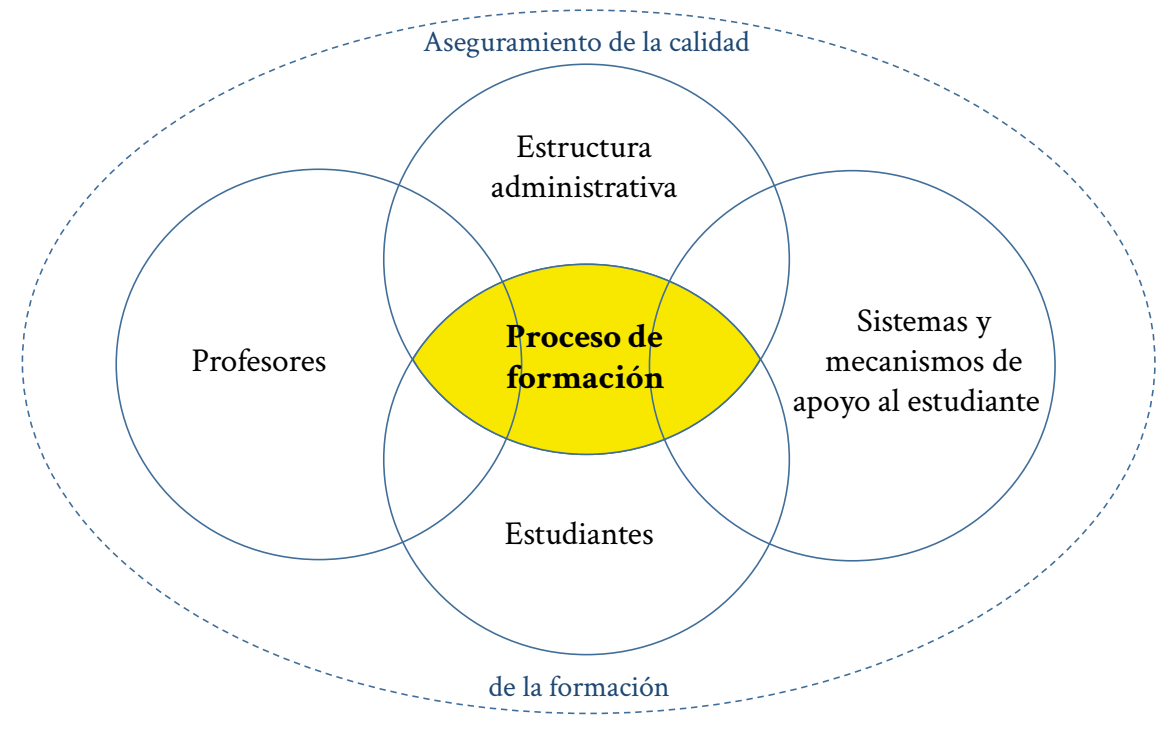

Figura 1. Elementos para asegurar la calidad del proceso de formación.

Fuente: elaboración propia.

\section{Propuesta de un nuevo marco de gestión de la calidad de la docencia}

\section{Implicancias y quehaceres de base de la actividad formativa}

En términos generales, la actividad docente debe estar organizada en un permanente desafío, de manera que cree interrogantes y discontinuidades dentro de un marco que busca sumar esfuerzos para que el estudiantado esté en el centro del proceso de aprendizaje (Hax y Ugarte, 2014). Un rasgo de esta interacción se relaciona con la actividad docente curricular, que cabe asumirla como una concepción que integra un conjunto de compromisos y actuaciones que se realizan tanto dentro del aula como fuera de ella. Está destinada a favorecer el aprendizaje de los estudiantes en cuanto al logro de los resultados de aprendizaje y las competencias definidas en el perfil de egreso, en un contexto institucional determinado. De esta manera, es posible indicar que la actividad docente-curricular implica, entre otros aspectos: 1) la coordinación, gestión y planificación de la docencia; 2) el despliegue de métodos de enseñanza; 3) las actividades de aprendizaje y evaluación; 4) la posterior revisión y mejora de las 
actuaciones realizadas; 5) la calidad de lo que se le ofrece al estudiantado, y 6) la implicación de la orientación curricular, que asume su valor por lo que aporta en la buena docencia universitaria.

\section{Los elementos que tributan hacia la articulación de una nueva propuesta de gestión docente}

Los elementos que se exponen en la figura 2 desprenden un único aspecto en común para los servicios de apoyo docente-formativo en el itinerario de la propuesta: la observación de que si se cristalizan dichos aspectos para el servicio del proceso formativo, se está ante una considerable relación articulada en la estructura organizacional, que se focaliza en una operación ordenada y que operacionaliza, en este nivel, múltiples tareas, funciones y responsabilidades en cada segmento. Esto tiene en cuenta un alcance para las condiciones de los macroprocesos en el emplazamiento por la cadena de valor de la institución.

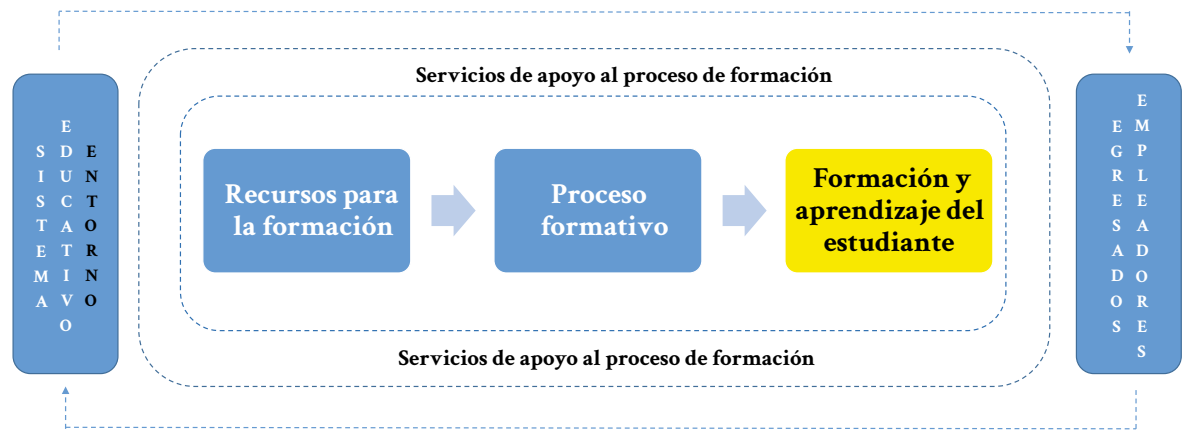

Figura 2. Servicios de apoyo docente para el proceso formativo.

Fuente: elaboración propia.

La idea clave que conforma esta perspectiva requiere situarse en los procesos indicados que dan origen a un núcleo de desarrollo que examina su propia matriz. Los macroprocesos que generan la posterior definición de una nueva estructura centrada en la gestión para la docencia se asumen en el contexto de una estructura contemporánea de la gestión, que se extrapola al contexto de las universidades. Es decir, la propuesta supone que la creación de conocimiento no solo tiene que involucrar un proceso administrativo, sino que debe reforzar de manera profunda el contexto organizacional. En este sentido, un motor de cambio es lo que lleva a asumir los 
lineamientos de un quehacer institucional de tipo hipertexto, que combina elementos de relaciones corporativas y relaciones dúctiles y flexibles que se recategorizan al tenor de la misma organización (Nonaka y Takeuchi, 1999), para el ámbito de la gestión de una docencia en contextos interconectados y concebidos, según las experiencias y valoraciones.

A partir de lo anterior, son tres los elementos que conforman una estructura devenida desde la misión y la visión institucionales, y con intercambios relacionales en diversos niveles, entre los que se destaca: la gestión del currículo — que incluye el desarrollo curricular-, la gestión del personal docente y la innovación de la docencia. En este modelo, a lo menos, se consideran tres factores relevantes como elementos del hipertexto en la gestión, atendiendo a su naturaleza. Estos factores se dan continuidad en el quehacer de manera interrelacionada y continua, cruzando diferentes niveles de acción para pasar de una mirada administrativa-burocrática de la gestión de la docencia a una realización de tipo articulada-combinada de índole autoorganizable. De este modo se propicia una oferta estratégica institucional de manera integral y flexible.

\section{La gestión del currículo}

Si la política de la docencia en una universidad se instala de manera vertical y jerarquizada, no podrá salir o trascender en la comunidad educativa, en el estudiantado, y menos en la comunidad territorial. Por extensión, no tendrá el impacto esperado ni las determinaciones de un adecuado posicionamiento como institución formadora de profesionales. La gestión del currículo debe instalarse para implementar una herramienta de mejora continua, de manera que atienda los análisis del perfil de ingreso y egreso y el quehacer formativo de las distintas especialidades. Además, debe agregar valor a la gestión institucional por medio de un marco de desarrollo curricular y pedagógico (Paricio, 2019).

Si el currículo se observa en la búsqueda de un impacto, la acción de este es más importante en la dinámica que entrega el desarrollo curricular, para lo cual atiende el conjunto de estrategias y acciones que emprende la universidad, destinadas a la obtención del logro de los distintos perfiles de egreso de las carreras y programas. A pesar del consenso general respecto de lo que se entiende por desarrollo curricular, en el sentido de implementar diseños y rediseños curriculares, sistematizando procesos y ahondando en los diseños de perfiles de egreso y en las revisiones de los planes de carrera, las dificultades inciden en que es necesario observar la temática de manera dual. Por un lado, se encuentran los factores de naturaleza administrativa y 
de clima organizacional y, por el otro, los factores tradicionales del currículo, teniendo en cuenta, de manera general, lo que indican Zhong y Sun (2018), que aluden a mantener una permanente revisión de los desarrollos curriculares dentro de la institución y asumir nuevas variables del hecho educativo en concordancia con la gestión administrativa de la categoría curricular.

Una vez consignados estos aspectos, se puede asumir en forma más precisa la caracterización de la acción pedagógica, que concentra una serie de relaciones de actividades: performance del docente, planteamientos de eficacia docente, estrategias que contribuyan al logro de los perfiles de egreso de las carreras o programas en los cuales se desempeña. En este contexto, los elementos que facilitan esa gestión son la capacitación y la preparación permanente de los docentes de la universidad, la promoción y el desarrollo de nuevas metodologías de enseñanza aprendizaje, la definición de estrategias que permitan la integración entre teoría y práctica y la focalización de la docencia en una coherencia constructiva para el proceso de aprendizaje.

Con base en estas evidencias, la institución esgrime razones para articular un trabajo que conlleva privilegiar un itinerario en el desarrollo curricular, que asume la presión por su cobertura, pero que también combina situaciones de naturaleza contextual y de gestión académica.

\section{La gestión del personal docente}

Si bien se observó el principio de un currículo para la transformación de las maneras de actuación académica que afectan positivamente al estudiantado en la universidad, con todos sus marcos de organización, planificación y detalle, asumiendo procesos en autonomía y confianza, también es posible advertir que junto con la renovación del currículo y de las metodologías educativas se busca identificar y validar la gestión del profesorado en un contexto que permita relevar la implementación de un modelo de enseñanza centrado en el logro de aprendizajes y la demostración de competencias (Villarroel y Bruna, 2017). Consecuentemente, las organizaciones universitarias tienen que dedicar más atención, priorización, selección y formación en su personal académico docente, ya que existe una particular condición de trabajo que, en la actualidad, y con el contexto de la pandemia como forma extensiva del quehacer, se encuentra en permanente transformación. Esto lleva a segmentar los diversos desempeños, las contrataciones y, por extensión, la realización de la docencia y la gestión académica. En este sentido, la propuesta en el modelo se caracteriza por gestionar una serie de tareas consistentes con la nueva asunción de la administración y la gestión por parte del personal docente, de acuerdo con el desarrollo de capacidades docentes 
responsables y efectivas en el desafío de ir construyendo un perfil del docente con orientación en la aplicación de un currículo con enfoque por competencias y centrado en la gestión de procesos de enseñanza-aprendizaje del estudiantado.

Esta gestión del personal docente, o académico, está vinculada, a su vez, con iniciativas internas que organizan la buena docencia, con impulsos de proyectos de desarrollo para la gestión de la docencia, con trabajos colaborativos en razón de las necesidades, acompañados de horas de dedicación a pesar del escenario de rigidez que se estila en las organizaciones universitarias estatales chilenas, en función de las dotaciones, el perfeccionamiento y el desarrollo, en general, de la cultura de tareas del personal docente en las universidades.

Otro aspecto importante que se aprecia en esta propuesta tiene que ver con la rendición de cuentas sobre una serie de variables y desempeños que miden la labor docente y que, gradualmente, se ha planteado la institución de manera particular. Esto implica un movimiento de gastos y de diversas fluctuaciones en el contexto de la organización y el sistema de educación superior chileno (Thedwall, 2008). Para los docentes en ejercicio, lo anterior se acompaña con un sistema de incentivos, los cuales solo están asociados a los estipendios por investigación en la docencia y a iniciativas internas que incorporan, de forma incipiente, ciertas dimensiones vinculadas a la evaluación del quehacer docente según el desempeño en la labor, con evidencias o demostrables.

\section{La innovación de la docencia}

La legitimidad de este factor es relevante para la gestión de la calidad formativa, puesto que es una acción que se construye en un marco de desarrollo académico en la línea de plantear dos cosas: por un lado, definir la docencia como prioridad de la gestión académica, asumiendo diversas experiencias con impacto educativo en las carreras o programas, proyectos y desarrollos docentes; por el otro, apuntar a que la docencia y la gestión académica contribuyan a la mejora continua de la docencia en pregrado y posgrado, aportando con líneas de investigación. Ese trabajo innovador se instala como una forma de liderazgo en una política nueva para las universidades estatales, con la idea de movilizar al profesorado a ejercer una buena docencia y de calidad, en un marco basado en la investigación, que transmita conocimiento y que facilite el aprendizaje (Jerez, Orsini y Hasbún, 2016).

Esta perspectiva de la gestión de calidad formativa tiene como propósito incentivar instancias para potenciar, mejorar y reproducir las buenas prácticas en docencia universitaria. Es uno de los aspectos más relevantes para encuadrar un marco de 
referencia de la calidad docente que se desarrolla en una universidad, ya que adiciona valor al quehacer y permite la creación y desarrollo de propuestas sobre investigación aplicada en docencia universitaria y/o la generación de instancias de intercambio académico interdisciplinario, las cuales facilitan la conformación de comunidades de aprendizaje. Por otra parte, la innovación en docencia tiene un compromiso con la mejora continua tanto en el modelo de buena práctica entre el profesorado como en la movilización hacia el desarrollo de los docentes por investigar en docencia y en gestión docente (Paricio, 2019). Ante la posibilidad de transformación, la institución estatal asume un gran desafío para los docentes, mediante el hecho de poner en marcha un programa periódico de formación e innovación en el profesorado de pregrado, y de manera incipiente en el de posgrado, en el marco de un programa que tiene como propósito la formación y la innovación para la adquisición y el perfeccionamiento de las competencias en diversas acciones docentes para el grupo de profesores interesados en el quehacer.

Los contenidos y la metodología del programa de innovación se orientan en la dirección de ir asumiendo, de manera dual, un proceso de cambio conceptual y metodológico que va acercando la profesionalización de la docencia a la institución, y va innovando en las actuaciones docentes dentro del aula, según las nuevas directrices de la universidad. De esta manera, tal actividad se encuentra conectada con la idea de Wood y Smith (2018) según la cual hay que fundamentar la innovación y la investigación en docencia sobre la base una concepción actualizada de esta, en un marco de desarrollo académico docente, con sus distintas visiones de mundo y metodologías de investigación. Esto incluye pensar en lo fundamental del quehacer: la reflexión acerca de los intereses de la investigación y la interacción crítica del proceso de estudio, que implica ampliar horizontes y proponer múltiples perspectivas colaborativas. De forma extensiva, la gestión de las iniciativas docentes como parte del proceso de formación e innovación en el profesorado es una modalidad que se constituye como un apoyo a los académicos de pregrado y posgrado para postular a convocatorias de proyectos docentes, los cuales tienen su correlación de gestión en el apoyo y seguimiento docente/académico de cada iniciativa y su escalamiento, posterior a productos de investigación que conllevan una presentación a congresos, foros, seminarios y, también, en el ámbito prospectivo, a artículos en revistas indexadas.

\section{La gestión de la información y de la administración en apoyo a la docencia}

Este tipo de gestión se define como una función emergente y de relevancia que busca potenciar el nivel de eficiencia de la universidad al incorporar la gestión del registro, 
los datos y, en general, la información para potenciar la labor docente, a fin de que se disponga de mayores antecedentes para tomar decisiones acertadas tanto en el aula como en las instancias intermedias de la gestión académica. El cumplimiento de evidencia en la relación directa con el valor que se hace de las informaciones centralizadas hace pensar en la complejidad y diversidad de la promoción por tener normas aceptadas y en permanente colaboración entre el área de la gestión y el área de la academia. En este cuadro general, también se asocian a la cadena de valor organizacional los componentes de recursos y de tecnologías de información para el desarrollo docente.

Las tareas recurrentes en ese aspecto son: 1) el control sobre la demanda, la actualización y la disponibilidad de bibliotecas y recursos informáticos, y cómo se añaden estos recursos informacionales a la docencia; 2) la administración estratégica de contenidos de información y herramientas para la docencia. En este ámbito se incorporan todos aquellos elementos que si bien no son el foco del proceso docente, son fundamentales para potenciar la calidad de la formación y la atención que recibe del estudiante. Estos aspectos son fundamentales para la evaluación que realiza el estudiante de la institución universitaria, y establecen relación con los servicios de matrícula, tesorería, registro curricular, inscripción de asignaturas, emisión de documentación relevantes para el estudiante, solicitud de beneficios estudiantiles, entre otros.

\section{Un nuevo entorno al servicio de la gestión de la docencia}

La gestión para fundamentar la cartografía de la docencia a escala institucional se orienta a la transversalización de la gestión de un proceso que debe atender la formación de personas, pero también debe garantizar evidencias de una enseñanza de calidad. Conforme a ello, lo que se busca a través de este enfoque es que esta labor agregue valor a la organización a través de las actividades que resultan del todo relevantes para conseguir ese propósito que, en el caso de la universidad, viene dado fundamentalmente por el desarrollo de la docencia y su innovación con un desarrollo del profesorado basado en la investigación, como parte de su quehacer integral y como parte de un marco ampliado de desarrollo docente. Esto va en línea con lo que proponen Jerez, Orsini y Hasbún (2016), al mencionar que la integración y la comprensión de las practicas docentes, que incluyen la gestión del currículo, las competencias disciplinares y pedagógicas y la efectividad del docente, entre otros aspectos, determinan los atributos generales de una docencia de calidad y su impacto en el aprendizaje del estudiantado. 
Conforme a lo anterior se ha de insistir en que los macroprocesos (García Solarte, 2009) son procesos globales o transversales a toda la organización ${ }^{6}$, y que al agruparlos la naturaleza del quehacer opera desde una relación directa con la función docente, acompañada de otros elementos que se relacionan con ella. Esta situación permite establecer una regla y una organización de la gestión a modo de lo que indica Yániz (2015) cuando comenta la importancia de transitar hacia nuevas significaciones de la docencia y a la integración de factores, esquemas o aprendizajes, como es lo que aparece, sintéticamente, en la figura 3.

\section{Macroprocesos de relación directa con la docencia}

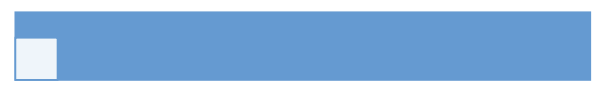

Gestión del currículum

Gestión de asuntos estudiantiles con incidencia en el currículum Innovación de la docencia

\section{Macroprocesos de apoyo} a la docencia

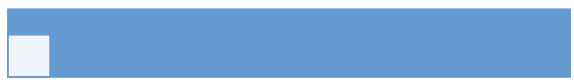

Gestión del personal docente Gestión de la información para la actividad docente Gestión de recursos materiales Gestión de aspectos administrativos de apoyo a la docencia

Figura 3. Macroprocesos, como procesos globales, en el ámbito de la docencia y de apoyo a la docencia. Fuente: elaboración propia.

La política implementada en la institución estatal se circunscribe dentro de las nuevas orientaciones de gestión que están asociadas a un aprendizaje social y académico, producto de modificaciones y ajustes que se han desarrollado en los últimos siete años en que se postula, según Villegas y Valderrama (2018), un mejoramiento transversal de la gestión institucional, que propende a cumplir una serie de objetivos estratégicos, el apoyo a la gestión académica y la asunción de seguimiento y monitoreo de múltiples acciones universitarias.

6 Este proceso permite determinar la creación de una dirección denominada de gestión y desarrollo curricular y otra de la docencia. Ambas propician la generación de estrategias y acciones que permitan la gestión del currículo, el aseguramiento de la planta académica, la gestión por investigar en docencia, el despliegue de las condiciones para que el profesorado se desarrolle en espacios de innovación y la planificación, implementación y monitoreo de los procesos de apoyo para el rediseño y la innovación curricular de carreras y programas, entre otros aspectos. 


\section{Resultados}

\section{La puesta en marcha de un sistema de aseguramiento de la calidad del diseño curricular: caso 1}

La adopción de un modelo centrado en el logro de aprendizajes y la demostración de competencias, según los fundamentos de Corvalán, Tardiff y Montero (2014), busca asegurar la calidad de la formación que desarrolla una institución, pero también robustece el foco de interés hacia los procesos de aseguramiento de la calidad, considerando los principios de la gestión en las universidades. Para lograr dicho propósito se puede orientar a definir un sistema en esa dirección, que se afinca en dos ejes fundamentales: definiciones estratégico-conceptuales y aspectos de apoyo técnicooperativo, que incorporan una serie de estrategias y acciones, conforme se indica en la figura 4, y que se recompensan en un sistema de trabajo y evaluación más continuo y permanente.

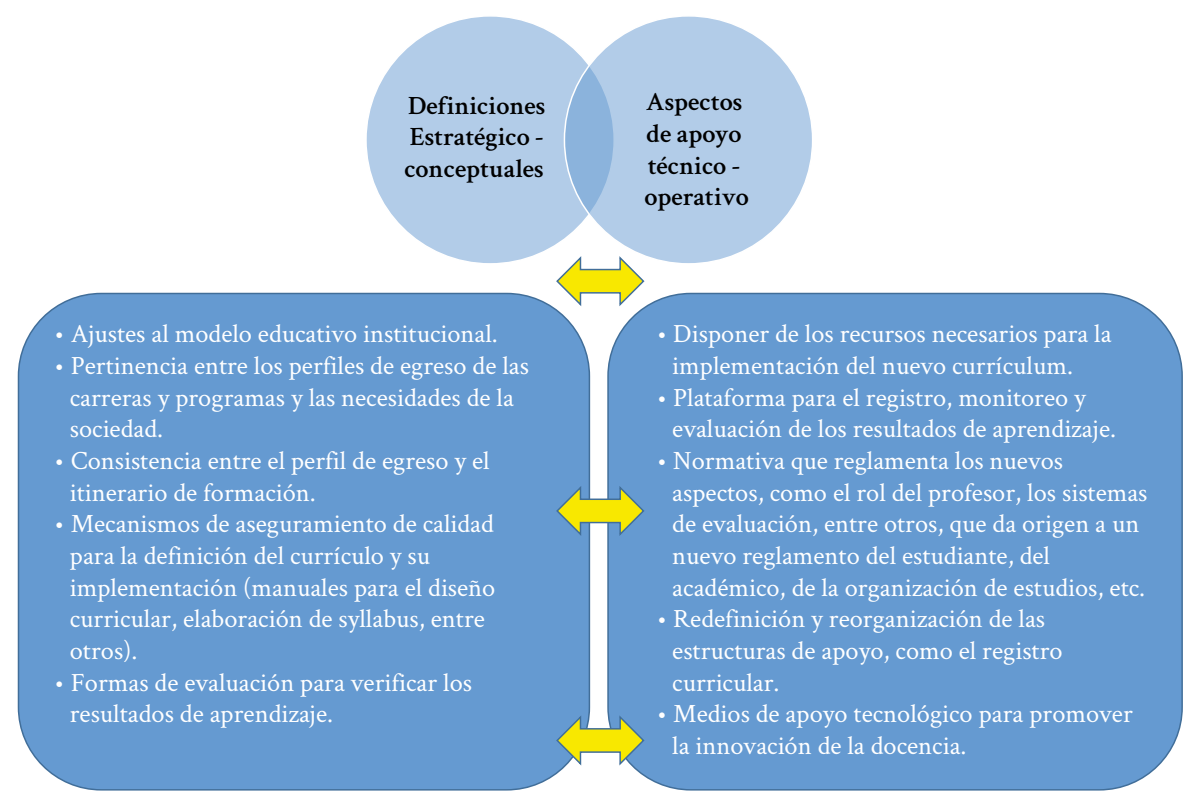

Figura 4. Definiciones estratégicas y de apoyo para el sistema de aseguramiento de la calidad del diseño curricular.

Fuente: elaboración propia. 
El propósito de la instalación del sistema de aseguramiento de la calidad del diseño curricular permitió, en términos generales, recoger las complejidades de su instalación en el profesorado y evaluar el proceso y las actividades que lo componen, con el objeto de identificar las oportunidades de mejora, planificar, diseñar e implementar los cambios requeridos, evaluar nuevamente el proceso, documentar los cambios y verificar que el diseño curricular de cada carrera y programa institucional se está realizando conforme se planeó, y con base en todos los mecanismos indicados en la figura 4, definidos como aspectos de apoyo técnico-operativo. La implementación del sistema, de acuerdo con la propuesta y el desarrollo escalado de su quehacer, arrojó el siguiente resultado: despliegue por ampliar el espacio del rediseño curricular en más del $80 \%$ de las carreras y programas de la institución. Esta cuestión introdujo el foco en los alcances de los planes de estudio, sancionados de manera administrativa a través de una oficialización, y haciendo una mención explícita al perfil de egreso, a las diversas competencias genéricas y específicas y a los resultados de aprendizaje.

El objetivo de esta preocupación, más allá de lo normativo, se traduce en la ventaja de un currículo que se va construyendo de manera colaborativa y teniendo en cuenta los resultados de aprendizaje, lo cual ha sido estudiado por algunos autores, como Caspersen, Frolich, Karlsen y Aamadot (2014). El beneficio para la institución está en que las carreras y programas han asumido la lógica del proceso de rediseño curricular en el marco de las indicaciones de la gestión participativa institucional, en la cual se han analizado los cumplimientos de las innovaciones y las participaciones docentes, a través de diversos comités (de innovación, rediseño, autoevaluación, entre otros), del compromiso administrativo del personal de apoyo y de los quehaceres que involucran cambios estructurales en el currículo, así como los ajustes y las complementaciones curriculares.

\section{Gestión e innovación de la docencia: los grupos de investigación y los proyectos docentes: caso 2}

El caso se refiere a una experiencia en la gestión en innovación de la docencia, en una búsqueda para acoger lo que significa avanzar en la calidad docente del profesorado como desafío profesional. Se establecieron dos ámbitos para el enfoque: los grupos de innovación e investigación y los proyectos docentes. Durante la última década, las instituciones universitarias han adoptado gradualmente la idea de que, en cuanto a consistencia y relevancia, la gestión docente se verá relevada a un mejor ámbito si se contribuye a crear, en el radio de las competencias docentes, una potencialidad institucional y docente para poner en marcha un programa formativo de sus cuadros 
académicos dentro de la institución, incluso más allá de las formas tradicionales de hacer docencia e investigación, puesto que lo que importa es construir un buen desempeño docente con transformaciones sostenibles en el tiempo (Carrasco y Flores, 2019). En este sentido, se analizaron los cambios de enfoque y las perspectivas respecto a los marcos para el desarrollo y la mejora docente. Esta exigencia permitió que los docentes comprendieran la importancia de investigar y publicar sus estudios o bien extender la difusión de sus trabajos en aula. La razón de base era una sola: la investigación educativa no constituía un área prioritaria de atención para los académicos y académicas que realizan docencia.

Todo esto implicó que desde 2015 comenzara un proceso gradual de irradiar una manera de conceptualizar la gestión en innovación docente, teniendo en cuenta que para ello los docentes deben definir sus performances para el desarrollo de estrategias de aprendizaje - ya sea realizando lecturas científicas, analizando bases de datos bibliográficas, desarrollando estudios de caso o en el contexto de la investigaciónacción, fomentando la escritura académica, amplificando redes colaborativas con otros docentes universitarios, entre otros aspectos- L La implementación de grupos organizados denominados núcleos de investigación en docencia considera múltiples actividades, entre las cuales se destaca el desarrollo de talleres dirigidos con base en las diversas asistencias técnicas que se centran en la metodología para la publicación de artículos científicos en revistas indexadas y en la evaluación de artículos por los pares.

Como una manera de potenciar el quehacer, se modificaron los instrumentos formales internos, lo que llevó a la creación de instancias de oficializaciones y, a la vez, se propició un grupo de trabajo para apoyar al profesorado desde una estructura organizada y con un plan anual de actividades. De forma paralela, con posterioridad a ese trabajo inicial, se agrupa de manera integral una forma tríadica para determinar el quehacer evidenciado por los primeros resultados, los cuales fueron piloto. Tales instancias permitieron conformar sesiones de seguimiento como una forma directa y contextualizada, a partir de un tema seleccionado y un proceso que se correlacionó con la participación de iniciativas concursales en materias docente. Esto se ajustó a resultados cada vez más innovadores y motivadores para el grupo docente, que relevaban el interés en la propuesta y la experiencia de intervención en gestión docente. 
Propuesta de un modelo de gestión para la docencia: experiencia de una universidad...

Tabla 1. Resultados globales de la actividad de los grupos de investigación en docencia.

\begin{tabular}{|l|l|}
\hline \multicolumn{1}{|c|}{ Actividades } & \multicolumn{1}{c|}{ Resultados } \\
\hline Grupos conformados & $\begin{array}{l}18 \text { grupos de innovación en docencia en distintas áreas } \\
\text { disciplinarias }\end{array}$ \\
\hline Docentes partícipes & 76 docentes en distinta condición contractual \\
\hline Facultades participantes & El total de las facultades de la institución \\
\hline Talleres y asistencias técnicas & 89 talleres y asistencias técnicas \\
\hline Publicaciones derivadas & 21 publicaciones en revistas indexadas \\
\hline Iniciativas docentes & 59 iniciativas \\
\hline
\end{tabular}

Fuente: elaboración propia

De la experiencia se comprende que la actividad de los grupos de profesores que se involucran en la gestión de la innovación en docencia responde a una práctica social que considera diversos aspectos de carácter individual e institucional. La respuesta a estas cuestiones se determina, por un lado, en que la formación teórica y práctica se sostiene en virtud del conjunto de experiencias de trabajos multidisciplinarios y esfuerzos estratégicos para incentivar la productividad de investigación $y$, por otro lado, en que los docentes se sitúan en una actividad reflexiva y social en el contexto de un cambio educativo. Los resultados, de esta manera, permiten escalar el interés de los grupos docentes interesados en la dinámica experiencial, situar los modos en que la institución de educación superior evidencia y organiza los alcances de esta iniciativa y la sistematización, cada vez más oportuna, en busca de una línea emergente de investigación multidisciplinaria en docencia. Este caso demuestra, desde la perspectiva del marco de desarrollo docente y profesional, que innovar en docencia y, a la vez, valorar el trabajo de investigación en docencia condensa y acumula efectivas y robustecidas prácticas docentes que se dirigen a líneas de acción que enfocan, de manera determinante, nuevos modos para afrontar la labor académica. De esta forma, se puede establecer definitivamente que hay una orientación a incrementar indicadores institucionales de la producción científica como una práctica que tiende a fortalecer una experiencia enfocada hacia el desarrollo de nuevos conocimientos en docencia. 


\section{Consideraciones en la implementación}

Un modelo de enseñanza centrado en el aprendizaje y la demostración de competencias tiene una serie de ventajas para la educación terciaria, ya que permite cautelar, con mayor énfasis, los resultados de aprendizajes de los estudiantes, lo cual da certeza del logro de los perfiles de egreso de las carreras y programas, y propicia una transición favorable entre el profesional recién egresado y el mundo del trabajo. Así mismo, permite una vinculación temprana del estudiante con la realidad profesional y las instancias formales en el currículo, determinando un proceso claro, previsible y en un marco de reflexión educativa (Zabalza y Zabalza, 2012) para el aseguramiento de la calidad. Focaliza su accionar en el estudiante, lo que facilita la implementación de dispositivos de nivelación y acompañamiento académico para alcanzar los aprendizajes esperados, entre muchas otras definiciones. Sin embargo, el desarrollo de acciones - por parte de la institución — en la línea de la adopción de un modelo formativo diferente está cargado también de dificultades, las cuales, para el caso particular, se han centrado en dos ejes: 1) la institución y la resistencia a un modelo de gestión innovador que hace hincapié en la calidad, 2) los docentes a través de la incorporación de sistemas de evaluación a los estudiantes que se focalizan en los aprendizajes y la necesidad de nuevos sistemas de evaluación docente.

\section{La resistencia al cambio}

Un enfoque de gestión en las instituciones de educación superior, basado en un modelo proactivo, que se anticipa al cambio y que obliga a su entorno a cambiar, no es una situación recurrente en las universidades estatales de Chile. Más bien impera un modelo de gestión que busca adaptarse al cambio de manera reactiva y, en algunos casos específicos, con cierta proactividad.

Las diversas instancias de la universidad, desde los niveles estratégico y táctico hasta el operativo, denotan resistencias como un efecto del nuevo enfoque y los procesos y procedimientos que este conlleva. En ese sentido, la gestión del cambio a escala institucional debe incorporarse como una modalidad para manejar los efectos de las nuevas orientaciones estratégicas de la universidad, en articulación con la cultura que impera en la institución y, en sí, con las personas que la componen, sobre todo si esa nueva estrategia implica el aprendizaje de nuevos comportamientos, habilidades y desempeños. En este contexto, requiere desarrollar herramientas para fortalecer los mecanismos de comunicación interna y la transparencia del actuar. 
Estos elementos suelen transformarse en aliados para que los miembros de la comunidad universitaria acepten de mejor forma el cambio organizacional y permanezcan comprometidos con las transiciones a pesar de las diferencias o disconformidades.

\section{La evaluación de los aprendizajes y la incorporación de indicadores}

Un cambio en el paradigma de formación y su adopción como modelo institucional, de alguna u otra manera, reviste cierto nivel de oposición por parte de los docentes, sobre todo si ello implica modificar la práctica docente y trasladar el protagonismo del proceso de enseñanza-aprendizaje desde el docente hacia el estudiante, afincado en la praxis más que en los referentes teóricos. A lo anterior se suman las necesidades de interdisciplinariedad que complejizan aún más los procesos evaluativos, al requerir mayor personalización para la medición de los aprendizajes alcanzados por los estudiantes.

La enseñanza y evaluación de aprendizajes transversales asociados a las competencias de formación integral declaradas por la universidad en su modelo educativo, tales como liderazgo, trabajo en equipo, comunicación, resolución de problemas, entre otras, son dificultades enormes si se considera, además, la responsabilidad en la valoración de dichos aprendizajes.

A todo esto se le añade otro factor que redunda en mayores dificultades, relacionado con la incorporación de indicadores para los procesos de evaluación, tanto del propio aprendizaje como de la gestión de la docencia, aun cuando permite darle objetividad y comparabilidad, personificando un lenguaje común que permite una medición estandarizada y una valoración en diversas magnitudes, siempre y cuando se tenga claridad absoluta sobre su propósito. El indicador que se debe utilizar en procesos que son de un carácter más bien cualitativo debe ser erigido con un criterio de utilidad y con una clara conceptualización que permita describir los efectos, resultados e impactos del proceso formativo.

\section{La implementación de un sistema de planificación y evaluación del desempeño académico}

La agilidad en la generación de nuevo conocimiento y la obsolescencia ineludible de él obligan a las instituciones de educación superior a reaccionar a dichos cambios. Por lo tanto, el profesor universitario que convive en esta nueva realidad requiere también de competencias y habilidades para desarrollar una docencia que impacte en el aprendizaje de los estudiantes y que permita la consolidación de un sistema educativo de calidad. Para ello, la incorporación de sistemas de planificación y evaluación docente 
que "permitan la obtención de información válida y confiable sobre las competencias y habilidades de enseñanza que posee un docente para favorecer y mediar el proceso de aprendizaje del alumno, así como también su actitud para crecer y fortalecerse profesionalmente a través de la evaluación” (Bruzual, Coburn, Hiller y Sanánez 2003, p. 2) resulta una tarea de trascendencia estratégica en el logro de los objetivos institucionales, referidos a la calidad organizacional y la calidad de su docencia.

El sistema integral de evaluación de la docencia descansa en la suscripción de un convenio anual de carácter vinculante entre el docente y su superior jerárquico, y además tiene en cuenta aspectos como el compromiso de realizar un conjunto de tareas que lleven a mejorar el aprendizaje de los estudiantes y la profesionalización de la labor; la existencia de un procedimiento conocido y estandarizado de cualificación de los logros alcanzados (criterios, rúbricas, categorías y puntajes), y la retroalimentación del proceso a través de sus efectos/impactos en el propio docente.

La implementación de este nuevo sistema, que incorpora la mirada desde la perspectiva de varios actores, entre los cuales se encuentran los estudiantes, el director de la unidad académica, la jefatura de carrera si no coincide con la autoridad anterior, además del propio docente, no favoreció la instauración del nuevo modelo, aun cuando su concepción y espíritu favoreciera la materialización de los preceptos institucionales.

\section{Conclusiones}

Hasta ahora, la implementación de un nuevo modelo de gestión de la docencia en una universidad estatal determina necesariamente la reestructuración y redefinición de los procesos académicos, con el propósito de asegurar la calidad de la formación que se proporciona a los estudiantes, de manera que las ofertas formativas institucionales respondan a los cambios que la sociedad demanda.

Con todo lo anterior, la política institucional ha logrado intervenir de manera central en el sentido de propiciar nuevas acciones en la comunidad académica, el profesorado y el estudiantado. De esta manera, ha considerado la calidad como una dinámica propia que acelera nuevas perspectivas y capacidades en el profesorado, a modo de lo que menciona Elmore (2010) en el más amplio sentido de la expresión. Se busca la calidad con el propósito de crear una formación de personas en el marco de una profesionalización del docente, que releve los desafíos asumidos por la sociedad de la información, la globalización y la hiperculturalidad. Desde ese hecho, 
y sabiendo que una institución gestiona cultura y calidad en el ámbito de una estrategia, también se considera lo que determinan el entorno y los diversos escenarios del trabajo docente, puesto que estos requieren, en el contexto inmediato de la pandemia, una revisión de la docencia y de los procesos curriculares, según lo indica el Informe Unesco/Iesalc (2020), puesto que los efectos de la crisis reciente y en desarrollo, en la educación terciaria, dejarán huella en los distintos actores educativos, particularmente por la ausencia de presencialidad en las actividades de formación.

Y la razón es muy simple: definir la misión institucional de una universidad debe estar en conexión con los propósitos de los actuales tiempos educativos, que requieren tener en cuenta diversidades en el aula, ejercicios profesionales diferentes en el mundo del trabajo, nuevas estructuras organizacionales en el quehacer y extensión de las interacciones, con miras a fortalecer un ejercicio reflexivo que atañe a este nuevo rol del profesorado y del estudiantado, para incorporar el enriquecimiento y el beneficio de una experiencia de aprendizaje, en la lógica de asumir actividades que incentiven la gestión de la docencia para todo el espacio formativo.

Sin embargo, esa transición hacia un nuevo modelo no es una tarea sencilla, ya que se presentan dificultades, la mayoría de ellas asociadas a la resistencia que se tiene frente a la innovación. Un elemento que se debe considerar, según Theurillat y Gareca (2015), es, por ejemplo, la tensión que ocurre entre los docentes que realizan la actividad docente y los que se ocupan de la investigación. Se pudo constatar en la implementación del cambio que no es posible lograr los propósitos planteados, si no existe el compromiso real de los actores involucrados — autoridades superiores, autoridades académicas, académicos, docentes y estudiantes - hacia el fin particular, que es mejorar los aprendizajes de los estudiantes y su preparación para enfrentar las demandas del mundo del trabajo.

Disminuir las resistencias hacia las nuevas directrices institucionales con planes de gestión del cambio resulta del todo relevante, ya que aun cuando se trata de innovaciones que nacen al alero del nivel superior de la organización, su materialización se efectúa en niveles que deben desarrollar un conocimiento más acabado respecto a los nuevos retos que debe impulsar una perspectiva más comprometida con la calidad de la educación. Finalmente, los tres factores implicados en la propuesta constituyen una oportunidad de la cual se puede sacar provecho, suponiendo un salto cualitativo en la política institucional, si hace evolucionar la triada de factores, y diferenciando la superación de las brechas entre lo que se formula de manera reproductiva y lo que se construye de forma distintiva en la lógica de contribución para la gestión de la docencia. 


\section{Sobre los autores}

Francisco Villegas Villegas. Doctor en Didáctica de las Letras, Artes y Humanidades por la Universidad de Barcelona. Máster en Investigación en Lengua y Literatura. Profesional de la Dirección de Aseguramiento de la Calidad de la Universidad de Antofagasta. Es docente en el Departamento de Ingeniería Civil Eléctrica de la Universidad de Antofagasta y profesor de didáctica de la lectura en la Escuela de Educación de la Universidad Católica del Norte (UCN). Publicaciones más recientes: "Los desafíos del convenio de armonización curricular en el contexto de la Universidad de Antofagasta: aseguramiento de la calidad formativa y de la gestión institucional” (2018); "Lógica de gobierno y de gestión en una universidad estatal de Chile" (2018); "Modelo de formación integral y sus principios orientadores: caso Universidad de Antofagasta” (2019).

Claudia Valderrama Hidalgo. Licenciada en Administración Pública. Administradora pública por la Universidad de Antofagasta, Chile. Actualmente es académica de la Facultad de Ciencias Sociales, Artes y Humanidades de la Universidad de Antofagasta. Máster en Educación, Universidad de Jaén. Es directora del Proyecto FIC-R Formación integral para la inserción laboral de estudiantes de la Universidad de Antofagasta y enseñanza media de la Región de Antofagasta. Ha sido directora de varios proyectos institucionales con fondos del Mineduc/Chile. Publicaciones más recientes: "Los desafíos del convenio de armonización curricular en el contexto de la Universidad de Antofagasta: aseguramiento de la calidad formativa y de la gestión institucional” (2018); "Lógica de gobierno y de gestión en una universidad estatal de Chile” (2018); “Modelo de formación integral y sus principios orientadores: caso Universidad de Antofagasta” (2019).

\section{Referencias}

Apple, M. W. (2018). ¿Puede la educación cambiar la sociedad? Santiago: Editorial Lom.

Bambrick-Santoyo, P. (2018). Las palancas del liderazgo. Una guía práctica para construir colegios exitosos. Santiago de Chile: Aptus Chile.

Bernasconi, A. (2015). La educación superior en Chile: transformación, desarrollo y crisis. Santiago de Chile: Ediciones uc.

Bruzual, D., Coburn, N., Hiller, C. y Sanánez, C. (2003). Evaluación del desempeño docente a través de un sistema en línea que permite obtener informes estadísticos inmediatos. Recuperado de http://www.niee.ufrgs.br/eventos/RIBIE/2008/pdf/evaluacion_desempeno_docente.pdf

Carrasco, A. y Flores, L. (2019). De la reforma a la transformación: capacidades, innovaciones y regulación de la educación chilena. Santiago: Ediciones UC.

Caspersen, J, Frolich, N., Karlsen, H. y Aamadot, P. O. (2014). Learning outcomes across disciplines and professions: measurement and interpretation. Quality in Higher Education, 2O(1). https://doi.org/10.1080/13538322.2014.904587 
Propuesta de un modelo de gestión para la docencia: experiencia de una universidad...

Cole, J. (2009). The Great American University. Nueva York: PublicAffairs.

Comisión Nacional de Acreditación. (2016). Nuevos criterios de acreditación para certificar carreras. Recuperado de https://www.cnachile.cl/noticias/paginas/NUEVOS-CRITERIOSPARA-PREGRADO.aspx

Consejo Nacional de Educación. (2017). Sistema Nacional de Aseguramiento de la Calidad de la Educación Superior. Recuperado de https://www.cned.cl/sistema-nacional-de-aseguramiento-de-la-calidad-de-la-educacion-superior-sinaces

Corvalán, O., Tardiff, J. y Montero, P. (Coords.) (2014). Manual para la innovación curricular universitaria basada en el desarrollo de competencias. Santiago de Chile: Ediciones Cipod.

Elmore, R. (2010). Mejorando la escuela desde la sala de clases. Santiago de Chile: Fundación Chile.

García Solarte, M. (2009). Los macro-procesos: un nuevo enfoque en el estudio de la gestión humana. Pensamiento y Gestión, (27), 162-200.

Hax, A. y Ugarte, J. J. (2014). Hacia la gran universidad chilena: un modelo de transformación estratégica. Santiago: Ediciones UC.

Hernández, C. y Guárate, A. (2017). Modelos didácticos para situaciones y contextos de aprendizaje. Madrid: Narcea.

Huerta, P. y Gaete, H. (2018). Análisis de la dependencia de la acreditación institucional: un estudio comparativo de universidades en Chile. Actualidades Investigativas en Educación, 18(1), 345-375. http://dx.doi.org/10.15517/AIE.V1811.31748

Jerez, O., Orsini, C. y Hasbún, B. (2016). Atributos de una docencia de calidad en la educación superior: una revisión sistemática. Estudios Pedagógicos, 42(3) 483-506.

Lemaitre, M. J., Maturana, M., Zenteno, E. y Alvarado, A. (2012). Cambios en la gestión institucional en universidades, a partir de la implementación del sistema nacional de aseguramiento de la calidad: la experiencia chilena. Calidad en la Educación, (36), 21-52. http:// dx.doi.org/10.4067/S0718-45652012000100001

Ley 20.129. (2020). Establece un Sistema Nacional de Aseguramiento de la Calidad de la Educación Superior. (Última versión). Ministerio de Educación. Santiago de Chile. Recuperado de https://www.leychile.cl/Navegar?idNorma=255323

Ley 21.091 (2018). Ley de Educación Superior. Santiago de Chile. Recuperado de https://www. leychile.cl/Navegar?idNorma=1118991

Morales, C. y Rueda, D. (2019). Aproximaciones teóricas a la calidad de la docencia universitaria. Revista Cubana de Educación Superior, 38(2), e.2.

Nonaka, I. y Takeuchi, H. (1999). La organización creadora de conocimiento. Ciudad de México: Oxford. 
Paricio, J. (2019). La calidad de lo que el estudiante hace: aprendizaje activo y constructivo. En Paricio, J., Fernández y A. Fernández. I. (Eds.). Cartografía de la buena docencia universitaria. Un marco para el desarrollo del profesorado basado en la investigación (pp. 57-88). Madrid: Narcea Ediciones.

Perkins, D. (2016). Educar para un mundo cambiante: ¿Quénecesitan aprender realmente los alumnos para el futuro? Madrid: Ediciones SM.

Pimienta, P. J. (2012). Las competencias en la docencia universitaria. México: Editorial Pearson.

Reimers, F. y Schleicher, A. (2020). Reporte marco para guiar una respuesta educativa a la pandemia 2020 del Covid-19. Recuperado de www.ciae.uchile.cl

Santelices, M. V., Catalán, X. y Horn, C. (2018). La educación superior en Chile: transformación, desarrollo y crisis. Santiago de Chile: Ediciones UC.

Thedwall, K. (2008). Nontenure-track faculty: Rising numbers, lost opportunities. New Directions for Higher Education, (143), 11-19.

Theurillat, D. y Gareca, B. (2015). Organización de docencia e investigación en universidades: una exploración al caso chileno. Revista Calidad en la Educación, (42), 120-160. http:// dx.doi.org/10.4067/S0718-45652015000100005

Unesco/Iesalc (2020). covid-19 y educación superior: de los efectos inmediatos al día después. Análisis de impactos, respuestas políticas y recomendaciones. Recuperado de http://www.iesalc. unesco.org/wp-content/uploads/2020/05/COVID-19-ES-130520.pdf

Véliz, D. y Bernasconi, A. (2019). Los académicos en la educación superior chilena: una profesión en transición. En: Carrasco, A. y Flores. L. (Eds.), De la reforma a la transformación: capacidades, innovaciones y regulación de la educación chilena. (pp. 323-347). Santiago: Ediciones UC.

Villarroel, V. y Bruna, D. (2017). Competencias pedagógicas que caracterizan a un docente universitario de excelencia: un estudio de caso que incorpora la perspectiva de docentes y estudiantes. Formación Universitaria, 10(4), 75-96.

Villegas, F. y Valderrama, C. (2018). Los desafíos del convenio de armonización curricular en el contexto de la Universidad de Antofagasta: aseguramiento de la calidad formativa y de la gestión institucional. En F. A. Ganga et ál. (Ed.), Investigaciones sobre gobernanza universitaria y formación ciudadana en educación (pp. 387-404). México: Editorial Fontamara Y UANL.

Wood, P. y Smith, J. (2018). Investigar en educación: conceptos básicosy metodología para desarrollar proyectos de investigación. Madrid: Narcea.

Yaniz, C. (2015). Las competencias genéricas como finalidad educativa. En: Villardón-Gallego (Ed.), Competencias genéricas de educación superior (pp. 13-24). Madrid: Narcea.

Yao, F. (2016). Los factores que influyen en la calidad de la educación. Revista Itinerario Educativo, 30(67) 217-225. https://doi.org/10.21500/0122753.2898 
Propuesta de un modelo de gestión para la docencia: experiencia de una universidad...

Zabalza, M. A. y Zabalza Cerderiña, M. A. (2012). Innovación y cambio en las instituciones educativas. Rosario, Argentina: Homo Sapiens.

Zhong, Z. y Sun, M. (2018). Rational examination of integrating "classics" into university general education curriculum: an empirical survey based on university. Educación y Sociedad de China, 51(1). https://doi.org/10.1080/10611932.2017.1411700 